\title{
Hyperbolicity of the graph of nonseparating multicurves
}

\author{
URSULA HAMENSTÄDT
}

\begin{abstract}
A nonseparating multicurve on a surface $S$ of genus $g \geq 2$ with $m \geq 0$ punctures is a multicurve $c$ so that $S-c$ is connected. For $k \geq 1$ define the graph $\mathcal{N C}(S, k)$ of nonseparating $k$-multicurves to be the graph whose vertices are nonseparating multicurves with $k$ components and where two such multicurves are connected by an edge of length one if they can be realized disjointly and differ by a single component. We show that if $k<g / 2+1$, then $\mathcal{N C}(S, k)$ is hyperbolic.
\end{abstract}

\section{Introduction}

The curve graph $\mathcal{C G}$ of an oriented surface $S$ of genus $g \geq 0$ with $m \geq 0$ punctures and $3 g-3+m \geq 2$ is the graph whose vertices are isotopy classes of essential (ie noncontractible and not homotopic into a puncture) simple closed curves on $S$. Two such curves are connected by an edge of length one if and only if they can be realized disjointly. The curve graph is a locally infinite $\delta$-hyperbolic geodesic metric space of infinite diameter (Masur and Minsky [6]) for a number $\delta>0$ not depending on the surface (Aougab [1], Bowditch [2], Clay, Rafi and Schleimer [3], Hensel, Przytycki and Webb [5]).

The mapping class group $\operatorname{Mod}(S)$ of all isotopy classes of orientation-preserving homeomorphisms of $S$ acts on $\mathcal{C G}$ as a group of simplicial isometries. This action is coarsely transitive, ie the quotient of $\mathcal{C G}$ under this action is a finite graph. Curve graphs and their geometric properties turned out to be an important tool for the investigation of the geometry of $\operatorname{Mod}(S)$ (Masur and Minsky [7]).

If the genus $g$ of $S$ is positive then for each $k \leq g$ we can define another $\operatorname{Mod}(S)-$ graph $\mathcal{N C}(S, k)$ as follows. Vertices of $\mathcal{N C}(S, k)$ are nonseparating $k$-multicurves, ie multicurves $v$ consisting of $k$ components such that $S-v$ is connected. Two such multicurves are connected by an edge of length one if they can be realized disjointly and differ by a single component. The mapping class group of $S$ acts coarsely transitively as a group of simplicial isometries on the graph of nonseparating $k$-multicurves. In fact, 
the action is transitive on vertices. Note that $\mathcal{N C}(S, 1)$ is just the complete subgraph of $\mathcal{C G}$ whose vertex set consists of all nonseparating simple closed curves in $S$.

The goal of this note is to show the following.

Theorem For $g \geq 2$ and $k<g / 2+1$ the graph $\mathcal{N C}(S, k)$ of nonseparating $k-$ multicurves is hyperbolic.

For the proof of the theorem, we adopt a strategy from Hamenstädt [4]. Namely, we begin by showing that for $g \geq 2$ the graph $\mathcal{N C}(S, 1)$ is hyperbolic. This is easy if $S$ has at most one puncture, in fact in this case the inclusion map $\mathcal{N C}(S, 1) \rightarrow \mathcal{C G}$ is a quasi-isometry (see Section 3). If $S$ has at least two punctures then this inclusion is not a quasi-isometry any more. In this case we apply a tool from [4]. This tool is also used in Section 4 to successively add components to the multicurve until the number $k<g / 2+1$ of components is reached.

We summarize the results from [4] which we need in Section 2. At the end of this note we give an example indicated to us by Tarik Aougab and Saul Schleimer which shows that the strict bound $g / 2+1$ for the number of components of the multicurve in the theorem is sharp.

Acknowledgements This work was carried out while the author visited the Institute for Pure and Applied Mathematics in Los Angeles. In a first version of this paper, I erroneously misstated the range of the number of components of multicurves for which the theorem is valid. I am grateful to Tarik Aougab and Saul Schleimer for pointing out this error to me. This work has been partially supported by the Hausdorff Center Bonn and ERC grant number 10160104.

\section{Hyperbolic extensions of hyperbolic graphs}

In this section we consider any (not necessarily locally finite) metric graph $(\mathcal{G}, d)$ (ie edges have length one). Let $\mathcal{C}$ be any finite, countable or empty index set. For a given family $\mathcal{H}=\left\{H_{c} \mid c \in \mathcal{C}\right\}$ of complete connected subgraphs of $\mathcal{G}$ define the $\mathcal{H}$-electrification of $\mathcal{G}$ to be the metric graph $\left(\mathcal{E} \mathcal{G}, d_{\mathcal{E}}\right)$ which is obtained from $\mathcal{G}$ by adding vertices and edges as follows.

For each $c \in \mathcal{C}$ there is a unique vertex $v_{c} \in \mathcal{E G}-\mathcal{G}$. This vertex is connected with each of the vertices of $H_{c}$ by a single edge of length one, and it is not connected with any other vertex. 
Definition 2.1 For a number $r>0$ the family $\mathcal{H}$ is called $r$-bounded if for $c \neq d \in \mathcal{C}$ the intersection $H_{c} \cap H_{d}$ has diameter at most $r$, where the diameter is taken with respect to the intrinsic path metric on $H_{c}$ and $H_{d}$.

A family which is $r$-bounded for some $r>0$ is simply called bounded.

In what follows, all parametrized paths $\gamma$ in $\mathcal{G}$ or $\mathcal{E} \mathcal{G}$ are supposed to be simplicial. This means that they are defined on a closed connected subset of the reals whose finite endpoints (if any) are integers. We require that the image of every integer is a vertex, and that the restriction to an integral interval $[k, k+1]$ either is a homeomorphism onto an edge, or it is constant. In particular, simplicial paths are continuous.

Call a simplicial path $\gamma$ in $\mathcal{E} \mathcal{G}$ efficient if for every $c \in \mathcal{C}$ we have $\gamma(k)=v_{c}$ for at most one integer $k$. Note that if $\gamma$ is an efficient simplicial path in $\mathcal{E} \mathcal{G}$ which passes through $\gamma(k)=v_{c}$ for some $c \in \mathcal{C}$ then $\gamma(k-1) \in H_{c}, \gamma(k+1) \in H_{c}$. This is true because the vertex $v_{c} \in \mathcal{E} \mathcal{G}$ is only connected with vertices in $H_{c}$ by an edge.

For a number $L>1$, an $L$-quasigeodesic in $\mathcal{E G}$ is a path $\gamma:[a, b] \rightarrow \mathcal{E G}$ such that for all $a \leq s<t \leq b$ we have

$$
|t-s| / L-L \leq d(\gamma(s), \gamma(t)) \leq L|t-s|+L .
$$

In slight deviation from this standard definition, we will require throughout that all quasigeodesics are simplicial, in particular, they are continuous. We will often but not always state this explicitly.

Definition 2.2 The family $\mathcal{H}$ has the bounded penetration property if it is $r$-bounded for some $r>0$ and if for every $L>0$ there is a number $p(L)>2 r$ with the following property. Let $\gamma$ be an efficient simplicial $L$-quasigeodesic in $\mathcal{E} \mathcal{G}$, let $c \in \mathcal{C}$ and let $k \in \mathbb{Z}$ be such that $\gamma(k)=v_{c}$. If the distance in $H_{c}$ between $\gamma(k-1)$ and $\gamma(k+1)$ is at least $p(L)$ then every efficient simplicial $L$-quasigeodesic $\gamma^{\prime}$ in $\mathcal{E} \mathcal{G}$ with the same endpoints as $\gamma$ passes through $v_{c}$. Moreover, if $k^{\prime} \in \mathbb{Z}$ is such that $\gamma^{\prime}\left(k^{\prime}\right)=v_{c}$ then the distance in $H_{c}$ between $\gamma(k-1), \gamma^{\prime}\left(k^{\prime}-1\right)$ and between $\gamma(k+1), \gamma^{\prime}\left(k^{\prime}+1\right)$ is at most $p(L)$.

Let $\mathcal{H}$ be as in Definition 2.2. Define an enlargement $\hat{\gamma}$ of an efficient simplicial $L$-quasigeodesic $\gamma:[0, n] \rightarrow \mathcal{E G}$ with endpoints $\gamma(0), \gamma(n) \in \mathcal{G}$ as follows. Let $0<k_{1}<\cdots<k_{s}<n$ be those points such that $\gamma\left(k_{i}\right)=v_{c_{i}}$ for some $c_{i} \in \mathcal{C}$. Then $\gamma\left(k_{i}-1\right), \gamma\left(k_{i}+1\right) \in H_{c_{i}}$. For each $i \leq s$ replace $\gamma\left[k_{i}-1, k_{i}+1\right]$ by a simplicial geodesic in the graph $H_{c_{i}}$ with the same endpoints. Note that since we require that the endpoints of $\gamma$ are vertices in $\mathcal{G}$, an enlargement of $\gamma$ is a path with the same endpoints. 
For a number $k>0$ define a subset $Z$ of the metric graph $\mathcal{G}$ to be $k$-quasiconvex if any geodesic with both endpoints in $Z$ is contained in the $k$-neighborhood of $Z$. In particular, up to perhaps increasing the number $k$, any two points in $Z$ can be connected in $Z$ by a (not necessarily continuous) path which is a $k$-quasigeodesic in $\mathcal{G}$.

In [4] the following is shown.

Theorem 2.3 Let $\mathcal{G}$ be a metric graph and let $\mathcal{H}=\left\{H_{c} \mid c \in \mathcal{C}\right\}$ be a bounded family of complete connected subgraphs of $\mathcal{G}$. Assume that the following conditions are satisfied.

(i) There is a number $\delta>0$ such that each of the graphs $H_{c}$ is $\delta$-hyperbolic.

(ii) The $\mathcal{H}$-electrification $\mathcal{E} \mathcal{G}$ of $\mathcal{G}$ is hyperbolic.

(iii) The family $\mathcal{H}$ has the bounded penetration property.

Then $\mathcal{G}$ is hyperbolic. There is a number $L>1$ such that enlargements of geodesics in $\mathcal{E} \mathcal{G}$ are $L$-quasigeodesics in $\mathcal{G}$. The subgraphs $H_{c}$ are uniformly quasiconvex.

In fact, although this was not stated explicitly, one obtains that the graph $\mathcal{G}$ is $\delta^{\prime}-$ hyperbolic for a number $\delta^{\prime}>0$ only depending on the hyperbolicity constant for $\mathcal{E} \mathcal{G}$, the common hyperbolicity constant $\delta$ for the subgraphs $H_{c}$ and the constants which enter in the bounded penetration property.

\section{Hyperbolicity of the graph of nonseparating curves}

In this section we consider an arbitrary surface $S$ of genus $g \geq 2$ with $m \geq 0$ punctures. Let $\mathcal{C G}$ be the curve graph of $S$ and let $\mathcal{N C}(S, 1)$ be the complete subgraph of $\mathcal{C G}$ whose vertex set consists of nonseparating curves. The goal of this section is to show the following proposition.

Proposition 3.1 The graph $\mathcal{N C}(S, 1)$ is hyperbolic.

Example If $S$ is a surface of genus $g=1$ then any two disjoint nonseparating simple closed curves in $S$ are homotopic after closing the punctures and the graph $\mathcal{N C}(S, 1)$ is not connected. 
Define a properly embedded connected incompressible subsurface $X$ of $S$ to be thick if the genus of $X$ equals $g$. This is equivalent to stating that each of the boundary circles of $X$ is separating in $S$ and that moreover there is no nonseparating simple closed curve in $S$ which is contained in $S-X$. Observe that the only thick subsurface of a surface $S$ with at most one puncture is $S$ itself.

If $X \subset S$ is thick then each component of $S-X$ is a bordered punctured sphere with connected boundary. If we collapse each boundary circle of $X$ to a puncture then we can view $X$ as a surface of finite type whose genus equals the genus of $S$. In particular, we can look at thick subsurfaces of $X$. However, the thick subsurfaces of $X$ are precisely the thick subsurfaces of $S$ which are contained in $X$.

For a thick subsurface $X$ of $S$ and for $p \geq 1$ define a graph $\mathcal{A}(X, p)$ as follows. The vertices of $\mathcal{A}(X, p)$ are nonseparating simple closed curves in $X$. Two such vertices $c, d$ are connected by an edge of length one if either they are disjoint or if they are both contained in a proper thick subsurface $Y$ of $X$ of Euler characteristic $\chi(X)+p$. Note that if $p \geq-\chi(X)-2 g+2$ then $\mathcal{A}(X, p)=\mathcal{N C}(X, 1)$.

Recall that for a number $L \geq 1$ two geodesic metric spaces $Y, Z$ are $L$-quasi-isometric if there is a map $F: Y \rightarrow Z$ so that

$$
d(x, y) / L-L \leq d(F x, F y) \leq L d(x, y)+L, \quad \forall x, y \in Y,
$$

and so that for all $z \in Z$ there is some $y \in Y$ with $d(F y, z) \leq L$. In general, quasiisometries are not continuous. A map $F: Y \rightarrow Z$ is called coarsely L-Lipschitz if $d(F x, F y) \leq L d(x, y)+L$ for all $x, y \in Y$.

Let $\mathcal{C G}(X)$ be the curve graph of $X$.

Lemma 3.2 For every thick subsurface $X$ of $S$ the vertex inclusion extends to a 2-quasi-isometry $\mathcal{A}(X, 1) \rightarrow \mathcal{C G}(X)$.

Proof Since two simple closed curves which are contained in a proper thick subsurface $Y$ of $X$ are disjoint from a boundary circle of $Y$ which is essential in $X$, the vertex inclusion extends to a coarsely 2 -Lipschitz map $\mathcal{A}(X, 1) \rightarrow \mathcal{C G}(X)$. Thus it suffices to show that the distance in $\mathcal{A}(X, 1)$ between any two nonseparating simple closed curves does not exceed twice their distance in $\mathcal{C G}(X)$.

To this end let $\gamma:[0, n] \rightarrow \mathcal{C G}(X)$ be a simplicial geodesic connecting two nonseparating simple closed curves $\gamma(0), \gamma(n)$. We construct first a simplicial geodesic $\tilde{\gamma}$ in $\mathcal{C G}(X)$ with the same endpoints such that for each $i$, the curve $\tilde{\gamma}(i)$ either is nonseparating or it decomposes $X$ into a thick subsurface of Euler characteristic $\chi(X)+1$ and a 
three-holed sphere. Call such a simple closed curve (with either of these two properties) admissible hereafter.

For the construction of $\tilde{\gamma}$ replace first each of the vertices $\gamma(2 i)$ with even parameter $0<2 i<n$ by an admissible curve. Namely, if $\gamma(2 i)$ is not admissible then $\gamma(2 i)$ decomposes $X$ into two surfaces $X_{1}, X_{2}$ which are different from three holed spheres.

If $\gamma(2 i-1), \gamma(2 i+1)$ are contained in distinct components of $X-\gamma(2 i)$ then they are disjoint and hence they are connected in $\mathcal{C G}(X)$ by an edge. This implies that we can shorten $\gamma$ with fixed endpoints. Since $\gamma$ is length-minimizing this is impossible.

Thus $\gamma(2 i-1), \gamma(2 i+1)$ are contained in the same component of $X-\gamma(2 i)$, say in $X_{1}$. Then $X_{2}=X-X_{1}$ either has positive genus and hence contains a nonseparating curve, or it is a sphere with at least four holes and contains an admissible separating curve. Thus there is an admissible curve $\tilde{\gamma}(2 i) \subset X_{2}$, and this curve is disjoint from $\gamma(2 i-1) \cup \gamma(2 i+1)$. Replace $\gamma(2 i)$ by $\tilde{\gamma}(2 i)$. This process leaves the points $\gamma(2 i+1)$ with odd parameter unchanged.

In the second step, replace with the same construction each of the points $\gamma(2 i+1)$ with odd parameter by an admissible curve. Let $\tilde{\gamma}:[0, n] \rightarrow \mathcal{C G}(X)$ be the resulting simplicial geodesic. The image of every vertex is admissible.

The geodesic $\tilde{\gamma}$ is now modified as follows. Replace each edge $\tilde{\gamma}[i, i+1]$ connecting two separating admissible simple closed curves $\tilde{\gamma}(i), \tilde{\gamma}(i+1)$ by an edge path in $\mathcal{C G}(X)$ of length 2 with the same endpoints so that the middle vertex is a nonseparating simple closed curve. This is possible because if $c_{1}, c_{2}$ are two disjoint separating admissible curves then $c_{1} \cup c_{2}$ is disjoint from some nonseparating simple closed curve in $X$. The length of the resulting path $\hat{\gamma}$ is at most twice the length of $\gamma$.

The path $\hat{\gamma}$ can be viewed as a path in $\mathcal{A}(X, 1)$ by simply erasing all vertices which are separating admissible simple closed curves. Namely, each such vertex $v$ is the boundary circle of a thick subsurface $Y$ of $X$ of Euler characteristic $\chi(X)+1$. The two adjacent vertices are nonseparating simple closed curves contained in $Y$. Thus by the definition of $\mathcal{A}(X, 1)$, these curves are connected in $\mathcal{A}(X, 1)$ by an edge. This shows that the endpoints of $\gamma$ are connected in $\mathcal{A}(X, 1)$ by a path whose length does not exceed twice the distance in $\mathcal{C G}(X)$ between the endpoints.

Since a surface with at most one puncture does not admit any proper thick subsurface we obtain the following as an immediate corollary.

Corollary 3.3 If $S$ has at most one puncture then the inclusion $\mathcal{N C}(S, 1) \rightarrow \mathcal{C G}$ is a 1-quasi-isometry. 
Write $\mathcal{A}(p)=\mathcal{A}(S, p)$. Our goal is to use Lemma 3.2 and induction on $p$ to show that $\mathcal{A}(p)$ is hyperbolic for all $p$. Since $\mathcal{A}(p)=\mathcal{N C}(S, 1)$ for $p \geq-\chi(S)-2 g+2$, this then shows Proposition 3.1.

Now let $p-1 \geq 1$ and let $X$ be a thick subsurface of $S$ such that $\chi(X)=\chi(S)+p-1$. Let $H_{X}$ be the complete subgraph of $\mathcal{A}(p)$ whose vertex set consists of all nonseparating simple closed curves which are contained in $X$. Let $\mathcal{H}=\left\{H_{X} \mid X\right\}$. Our goal is to apply Theorem 2.3 to the graph $\mathcal{A}(p)$ and its $\mathcal{H}$-electrification. The next easy observation is the basic setup for the induction step.

Lemma 3.4 We have that $\mathcal{A}(p-1)$ is 2-quasi-isometric to the $\mathcal{H}$-electrification of $\mathcal{A}(p)$.

Proof Let $\mathcal{E}$ be the $\mathcal{H}$-electrification of $\mathcal{A}(p)$. Let $c, d$ be any two simple closed curves which are connected in $\mathcal{A}(p-1)$ by an edge. Then either $c, d$ are disjoint and hence connected in $\mathcal{A}(p)$ by an edge, or $c, d$ are contained in a thick subsurface $X$ of $S$ of Euler characteristic $\chi(X)=\chi(S)+p-1$. Thus $c, d$ are vertices in $H_{X}$ and hence the distance between $c, d$ in $\mathcal{E}$ is at most two. This shows that the vertex inclusion $\mathcal{A}(p-1) \rightarrow \mathcal{E}$ is 2 -Lipschitz.

That this is in fact a 2 -quasi-isometry follows from the observation that $\mathcal{A}(p)$ is obtained from $\mathcal{A}(p-1)$ by deleting some edges. Moreover, the endpoints of an embedded simplicial path in $\mathcal{E}$ of length 2 whose midpoint is a special vertex not contained in $\mathcal{A}(p)$ are nonseparating simple closed curves which are contained in a thick subsurface $X$ of $S$ of Euler characteristic $\chi(S)+p-1$, and hence they are connected by an edge in $\mathcal{A}(p-1)$.

Our goal is now to check that the family $\mathcal{H}=\left\{H_{X} \mid X\right\}$ has the properties stated in Theorem 2.3. The following lemma together with Lemma 3.2 implies that the graphs $H_{X}$ are $\delta$-hyperbolic for a universal constant $\delta>0$.

Lemma 3.5 We have that $H_{X}$ is isometric to $\mathcal{A}(X, 1)$.

Proof Let $X$ be a thick subsurface of $S$ of Euler characteristic $\chi(S)+p-1$. If $c_{1}, c_{2}$ are two nonseparating simple closed curves contained in $X$ then $c_{1}, c_{2}$ are connected in $\mathcal{A}(p)$ by an edge if either $c_{1}, c_{2}$ are disjoint or if $c_{1}, c_{2}$ are contained in a thick subsurface $X_{0}$ of $S$ of Euler characteristic $\chi\left(X_{0}\right)=\chi(S)+p=\chi(X)+1$.

Now the thick subsurface $X_{0}$ can be chosen to be contained in $X$. Namely, any thick subsurface of $S$ of Euler characteristic $\chi(S)+\ell(\ell \geq 0)$ can be described as the complement in $S$ of a small neighborhood of an embedded forest in $S$ (ie an 
embedded, possibly disconnected graph with no cycles) with $\ell$ edges whose vertices are the punctures of $S$.

Assume for the moment that there is a forest $G$ defining $X$ which is the union of a connected component $\widehat{G}$ and isolated points. Then $\widehat{G}$ has precisely $p$ vertices and $p-1$ edges, where $p-1=\chi(X)-\chi(S)$. Since the graph $G_{0}$ defining $X_{0}$ has $p$ edges, there is at least one puncture $x$ of $S$ which is not contained in $\widehat{G}$ and which is the endpoint of an edge $e$ of $G_{0}$. If, up to homotopy with fixed endpoints, $e$ intersects $\widehat{G}$ at most at the second endpoint then the complement of a small neighborhood of $\widehat{G} \cup e$ is a thick subsurface $Y$ of $X$ of Euler characteristic $\chi(S)+p$ which contains $c_{1} \cup c_{2}$. This is what we wanted to show.

If $e$ intersects $\widehat{G}$ in an interior point which can not be removed with a homotopy of $e$ with fixed endpoints, let $e_{0}$ be the subarc of $e$ with endpoints $x$ and the first intersection point with $\widehat{G}$. Concatenation of $e_{0}$ with a subarc of an edge of $\widehat{G}$ and modification of the resulting arc with a small homotopy with fixed endpoints yields an embedded arc $\hat{e}$ in $S$ whose interior is disjoint from the interior of $\widehat{G}$, so that $\widehat{G} \cup \widehat{e}$ defines a thick subsurface $Y$ of $X$ of Euler characteristic $\chi(S)+p$ disjoint from $c_{1} \cup c_{2}$.

The general case is treated in the same way. Namely, there is at least one edge $e$ of $G_{0}$ which connects two distinct connected components of $G$, and the argument above can be applied to the edge $e$.

As a consequence, $c_{1}, c_{2}$ are connected by an edge in $\mathcal{A}(X, 1)$, which is what we wanted to show.

Lemma 3.6 The family of subgraphs $H_{X}$ of $\mathcal{A}(p)$, where $X$ runs through the thick subsurfaces of $S$ of Euler characteristic $\chi(S)+p-1$, is bounded.

Proof Let $X, Y$ be two thick subsurfaces of $S$ of Euler characteristic $\chi(S)+p-1$. If $X \neq Y$ then up to homotopy, $X \cap Y$ is a (possibly disconnected) subsurface of $X$ whose Euler characteristic is strictly bigger than the Euler characteristic of $X$. In particular, the diameter in the curve graph of $X$ of the set of simple closed curves contained in $X \cap Y$ is uniformly bounded. Thus the lemma follows from Lemmas 3.2 and 3.5 .

The proof of the bounded penetration property is more involved. To this end recall from [7] that for every proper connected subsurface $X$ of $S$ there is a subsurface projection $\pi_{X}$ of $\mathcal{C G}$ into the subsets of the arc and curve graph of $X$. This projection associates to a simple closed curve $c$ in $S$ which is not disjoint from $X$ the intersection 
components $\pi_{X}(c)$ of $c$ with $X$, viewed as a subset of the arc and curve graph of $X$. The diameter of the image is at most one. If $c$ is disjoint from $X$ then this projection is empty. The arc and curve graph of $X$ is 2-quasi-isometric to the curve graph of $X$ (see [7]).

Recall that every vertex of any of the graphs $\mathcal{A}(p)(p \geq 1)$ is a nonseparating simple closed curve in $S$. By definition of a thick subsurface of $S$, for any such curve $c$ and every thick subsurface $Z$ of $S$ we have $\pi_{Z}(c) \neq \varnothing$. This fact will be used throughout the remainder of this section.

We need the following result from [7] (in the version formulated in [4, Lemma 6.5]).

Proposition 3.7 For every number $L>1$ there is a number $\xi(L)>0$ with the following property. Let $Y$ be a proper connected subsurface of $S$ and let $\gamma$ be a simplicial path in $\mathcal{C G}$ which is an $L$-quasigeodesic. If $\pi_{Y}(v) \neq \varnothing$ for every vertex $v$ on $\gamma$ then

$$
\operatorname{diam} \pi_{Y}(\gamma)<\xi(L) .
$$

If $\gamma:[0, n] \rightarrow \mathcal{A}(S, 1)$ is any geodesic then for all $j$, the curves $\gamma(j)$ and $\gamma(j+1)$ either are disjoint and hence connected in $\mathcal{C G}$ by an edge, or they are contained in a common thick subsurface $Y$ of $S$ of Euler characteristic $\chi(S)+1$. In the second case replace the edge $\gamma[j, j+1]$ by an edge path in $\mathcal{C G}$ of length two connecting the same endpoints which passes through an essential simple closed curve in the complement of $Y$. We call $\tilde{\gamma}$ a canonical modification of $\gamma$. By Lemma 3.2 and its proof, $\tilde{\gamma}$ is a simplicial path in $\mathcal{C G}$ which is a 2 -quasigeodesic.

We now define a family of geodesics in $\mathcal{A}(S, 1)$ which serve as substitutes for the tight geodesics as introduced in [7]. Namely, for numbers $\kappa>0, p \geq 1$, define a simplicial path $\zeta:[0, k] \rightarrow \mathcal{A}(S, 1)$ to be $(\kappa, p)$-good if the following holds true. Let $X \subset S$ be any thick subsurface of Euler characteristic $\chi(X) \geq \chi(S)+p$; then there is a number $u=u(X) \in[0, k)$ with the following property.

(i) For every $j \leq u$, $\operatorname{diam}\left(\pi_{X}(\zeta(0) \cup \zeta(j))\right) \leq \kappa$.

(ii) For every $j>u, \operatorname{diam}\left(\pi_{X}(\zeta(j) \cup \zeta(k))\right) \leq \kappa$.

Thus in a good simplicial path, big subsurface projections into thick subsurfaces can be explicitly localized.

We use Proposition 3.7 to show the following lemma.

Lemma 3.8 There is a number $\kappa_{1}>0$ such that any two vertices in $\mathcal{A}(S, 1)$ can be connected by a $\left(\kappa_{1}, 1\right)$-good geodesic. 
Proof Let $c_{1}, c_{2}$ be nonseparating simple closed curves and let $\gamma:[0, k] \rightarrow \mathcal{A}(S, 1)$ be a simplicial geodesic connecting $c_{1}$ to $c_{2}$, with canonical modification $\tilde{\gamma}:[0, \widetilde{k}] \rightarrow \mathcal{C G}$. Let $\ell_{1}<\cdots<\ell_{s}$ be such that for each $i$ the curves $\tilde{\gamma}\left(\ell_{i}\right), \tilde{\gamma}\left(\ell_{i}+2\right)$, are both separating and such that the subsurface of $S$ filled by $\tilde{\gamma}\left(\ell_{i}\right) \cup \tilde{\gamma}\left(\ell_{i}+2\right)$ (ie the smallest subsurface of $S$ which contains $\left.\tilde{\gamma}\left(\ell_{i}\right) \cup \tilde{\gamma}\left(\ell_{i}+2\right)\right)$ is a holed sphere whose complement $Z$ in $S$ is thick (we may have $s=0$, ie there may not be such a pair of vertices). Then $\tilde{\gamma}\left(\ell_{i}+1\right)$ is a nonseparating simple closed curve contained in $Z$. Let $\tilde{\gamma}_{1}\left(\ell_{i}+1\right)$ be a nonseparating simple closed curve contained in $Z$ which is contained in the 1 -neighborhood of the subsurface projection $\pi_{Z}\left(c_{2}\right)$ of $c_{2}$. That the subsurface projection $\pi_{Z}\left(c_{2}\right)$ is not empty follows since $c_{2}$ is nonseparating and hence can not be contained in $S-Z$.

Replace $\tilde{\gamma}\left(\ell_{i}+1\right)$ by $\tilde{\gamma}_{1}\left(\ell_{i}+1\right)$. The simplicial path $\tilde{\gamma}_{1}$ constructed in this way is a canonical modification of a geodesic $\gamma_{1}$ in $\mathcal{A}(S, 1)$ connecting $c_{1}$ to $c_{2}$. We claim that $\gamma_{1}$ is a $(\xi(2), 1)$-good geodesic in $\mathcal{A}(S, 1)$ where $\xi(2)>0$ is as in Proposition 3.7.

Namely, if $Z$ is an arbitrary thick subsurface of $S$ then since $\gamma_{1}$ is a geodesic in $\mathcal{A}(S, 1)$, there are at most two parameters $k, k+\iota$ (here $\iota=0$ or $\iota=2$ ) such that $\tilde{\gamma}_{1}(k), \tilde{\gamma}_{1}(k+\iota)$ are disjoint from $Z$. Since $\tilde{\gamma}_{1}$ is a 2 -quasigeodesic in $\mathcal{C G}$, if there is at most one such point (which is in particular the case if the Euler characteristic of $Z$ equals $\chi(S)+1$, ie if there is a unique essential curve disjoint from $Z$ ) then the properties (i), (ii) for $Z$ with $\kappa=\xi(2)$ are immediate from Lemma 3.2 and Proposition 3.7. Otherwise the property follows from the construction of $\gamma_{1}$ and the fact that for subsurfaces $X \subset Y \subset S$ and any simple closed curve $c$ we have $\pi_{X}(c)=\pi_{X}\left(\pi_{Y}(c)\right)$ (with a small abuse of notation).

We use Proposition 3.7 and Lemma 3.8 to define a level-p hierarchy path in $\mathcal{A}(p)$ connecting two nonseparating simple closed curves $c_{1}, c_{2}$ as follows. The starting point is a $\left(\kappa_{1}, 1\right)$-good geodesic $\gamma:[0, k] \rightarrow \mathcal{A}(S, 1)$. For any $j$ such that the curves $\gamma(j), \gamma(j+1)$ are not disjoint there is a thick subsurface $Y_{j}$ of Euler characteristic $\chi\left(Y_{j}\right)=\chi(S)+1$ so that $\gamma(j), \gamma(j+1) \subset Y_{j}$. Replace the edge $\gamma[j, j+1]$ by a simplicial $\left(\kappa_{1}, 1\right)$-good geodesic in $\mathcal{A}\left(Y_{j}, 1\right)$ with the same endpoints. The resulting path is an edge-path in the subgraph $\mathcal{A}(2)$ of $\mathcal{A}(1)$. Proceed inductively and construct in $p$ such steps a simplicial path in $\mathcal{A}(p) \subset \mathcal{A}(1)$ connecting $c_{1}$ to $c_{2}$, which we call a level-p hierarchy path.

Lemma 3.9 For every $p \geq 1$ there is a number $\kappa_{p}>0$ such that a level- $p$ hierarchy path in $\mathcal{A}(p)$ is $\left(\kappa_{p}, p\right)$-good.

Proof We proceed by induction on $p$. The case $p=1$ follows from the definition of a hierarchy path and Lemma 3.8. Thus assume that the lemma holds true for all $p-1 \geq 1$. 
Let $\gamma:[0, n] \rightarrow \mathcal{A}(p)$ be a level- $p$ hierarchy path. The construction of $\gamma$ is as follows. There is a level- $(p-1)$ hierarchy path $\zeta:[0, s] \rightarrow \mathcal{A}(p-1)$, and there are numbers $0 \leq \tau_{1}<\cdots<\tau_{q}<s$ such that for each $i$, the edge $\zeta\left[\tau_{i}, \tau_{i}+1\right]$ connects two nonseparating simple closed curves which are contained in a thick subsurface $Z_{i}$ of $S$ of Euler characteristic $\chi(S)+p-1$. For $\ell \notin\left\{\tau_{1}, \ldots, \tau_{q}\right\}$, the simple closed curves $\zeta(\ell), \zeta(\ell+1)$ are disjoint. The hierarchy path $\gamma$ is obtained from $\zeta$ by replacing each of the edges $\zeta\left[\tau_{i}, \tau_{i}+1\right]$ by a $\left(\kappa_{1}, 1\right)$-good geodesic in $\mathcal{A}\left(Z_{i}, 1\right)$ with the same endpoints.

By induction hypothesis, $\zeta$ is $\left(\kappa_{p-1}, p-1\right)$-good for a number $\kappa_{p-1}>1$ not depending on $\zeta$. Thus for any thick subsurface $Z$ of $S$ of Euler characteristic $\chi(Z) \geq \chi(S)+p$ there is a number $u \in[0, s]$ such that for all $j \leq u$, $\operatorname{diam}\left(\pi_{Z}(\zeta(0) \cup \zeta(j))\right) \leq \kappa_{p-1}$ and similarly for $j \geq u+1$.

Let now $i>0$ be such that $\tau_{i}<u$. There is a subarc $\rho$ of $\gamma$ which is a $\left(\kappa_{1}, 1\right)$-good geodesic in $\mathcal{A}\left(Z_{i}, 1\right)$ connecting $\zeta\left(\tau_{i}\right)$ to $\zeta\left(\tau_{i}+1\right)$. By the definition of a $\left(\kappa_{1}, 1\right)$-good geodesic in $\mathcal{A}\left(Z_{i}, 1\right)$, since

$$
\begin{aligned}
\operatorname{diam}\left(\pi_{Z}\left(\zeta\left(\tau_{i}\right) \cup \zeta\left(\tau_{i}+1\right)\right)\right) & \\
& \leq \operatorname{diam}\left(\pi_{Z}\left(\zeta(0) \cup \zeta\left(\tau_{i}\right)\right)\right)+\operatorname{diam}\left(\pi_{Z}\left(\zeta(0) \cup \zeta\left(\tau_{i}+1\right)\right)\right) \leq 2 \kappa_{p-1},
\end{aligned}
$$

for each vertex $\rho(t)$ on the geodesic $\rho$ the diameter of the subsurface projection $\pi_{Z}\left(\zeta\left(\tau_{i}\right) \cup \rho(t)\right)$ does not exceed $2 \kappa_{p-1}+\kappa_{1}$. Then for each $t$ we have

$$
\operatorname{diam}\left(\pi_{Z}(\zeta(0) \cup \rho(t))\right) \leq 3 \kappa_{p-1}+\kappa_{1}=\kappa_{p} .
$$

This argument is also valid for $\tau_{i}>u$.

Finally, if $\tau_{i}=u$ then we can apply the same reasoning as before to the $\kappa_{1}$-good geodesic $\rho$ and obtain the statement of the lemma.

Proof of Proposition 3.1 By Corollary 3.3, if $S$ has at most one puncture then the inclusion $\mathcal{N C}(S, 1) \rightarrow \mathcal{C G}$ is a quasi-isometry.

If the number of punctures is at least two then we show by induction on $p$ the following.

(a) The graph $\mathcal{A}(p)$ is hyperbolic.

(b) Level- $p$ hierarchy paths are uniform quasigeodesics in $\mathcal{A}(p)$.

(c) For every $L>1$ there is a number $\xi(L, p)>0$ with the following property. Let $\gamma$ be a simplicial path in $\mathcal{A}(p)$ which is an $L$-quasigeodesic, and let $\tilde{\gamma}$ be a canonical modification of $\gamma$. If $Y$ is a thick subsurface of $S$ of Euler characteristic $\chi(Y) \geq \chi(S)+p$, and if $\pi_{Y}(v) \neq \varnothing$ for every vertex $v$ on $\tilde{\gamma}$ then $\operatorname{diam} \pi_{Y}(\gamma)<\xi(L, p)$. 
The case $p=1$ follows from Lemma 3.2, Proposition 3.7 and the definition of a canonical modification of a simplicial path in $\mathcal{A}(S, 1)$. Assume that the claim holds true for $p-1 \geq 1$.

For a thick subsurface $X$ of Euler characteristic $\chi(X)=\chi(S)+p-1$ let as before $H_{X}$ be the complete subgraph of $\mathcal{A}(p)$ whose vertex set consists of all nonseparating simple closed curves contained in $X$, and let $\mathcal{H}=\left\{H_{X} \mid X\right\}$. By Lemma 3.4, $\mathcal{A}(p-1)$ is 2 -quasi-isometric to the $\mathcal{H}$-electrification of $\mathcal{A}(p)$. Moreover by construction, level- $p$ hierarchy paths are enlargements of level- $(p-1)$ hierarchy paths. Therefore by the induction hypothesis, to establish properties (a), (b) above for $p$ it suffices to show that the family $\mathcal{H}$ is bounded and satisfies the assumptions (1), (3) in the statement of Theorem 2.3.

Lemma 3.6 shows that the family $\mathcal{H}=\left\{H_{X} \mid X\right\}$ is bounded.

By Lemma 3.5, $H_{X}$ is isometric to $\mathcal{A}(X, 1)$ and hence by Lemma 3.2, $H_{X}$ is $\delta-$ hyperbolic for a number $\delta>0$ not depending on $X$. The bounded penetration property for $\mathcal{H}$ follows from property (c) above, applied to thick subsurfaces of Euler characteristic $\chi(S)+p$ and quasigeodesics in $\mathcal{A}(p-1)$ (compare [4]). Thus by Theorem 2.3 and the induction hypothesis, $\mathcal{A}(p)$ is hyperbolic, and level- $p$ hierarchy paths are uniform quasigeodesics in $\mathcal{A}(p)$.

We are left with verifying property (c) above for $\mathcal{A}(p)$. By Lemma 3.9, this property holds true for level- $p$ hierarchy paths with the number $\kappa_{p}>0$ replacing $\xi(L, p)$. The argument in the proof of [4, Lemma 6.5] then yields this property for an arbitrary $L$-quasigeodesic in $\mathcal{A}(p)$ for a suitable number $\xi(L, p)>0$.

Namely, by hyperbolicity, for every $L>1$ there is a number $n(L)>1$ so that for every $L$-quasigeodesic $\eta:[0, k] \rightarrow \mathcal{A}(p)$ of finite length, the Hausdorff distance between the image of $\eta$ and the image of a level- $p$ hierarchy path $\gamma$ with the same endpoints does not exceed $n(L)$.

Let $Y \subset S$ be a thick subsurface of Euler characteristic $\chi(Y) \geq \chi(S)+p$. Assume that

$$
\operatorname{diam}\left(\pi_{Y}(\eta(0) \cup \eta(k))\right) \geq 2 \kappa_{p}+L(4 n(L)+10)=\xi(L, p) .
$$

By the properties of level- $p$ hierarchy paths, if $\tilde{\gamma}$ denotes a canonical modification of $\gamma$ then there is some $u \in \mathbb{Z}$ so that $\tilde{\gamma}(u) \in A$ where $A \subset \mathcal{C G}$ is the set of all curves which are disjoint from $Y$.

By the choice of $n(L)$, the quasigeodesic $\eta$ passes through the $n(L)$-neighborhood of $Y$. By this we mean that there is a vertex $x$ on $\eta$ and a simplicial path in $\mathcal{A}(p)$ of length at most $n(L)$ which connects $x$ to a nonseparating simple closed curve contained in $Y$. 
Let $s_{0}+1 \leq t_{0}-1$ be the smallest and biggest number, respectively, so that $\eta\left(s_{0}+1\right)$, $\eta\left(t_{0}-1\right)$ are contained in the $n(L)$-neighborhood of $Y$ in the sense defined in the previous paragraph. The distance in $\mathcal{A}(p)$ between $\eta\left(s_{0}\right)$ and $\eta\left(t_{0}\right)$ does not exceed $2 n(L)+3$.

A level- $p$ hierarchy path connecting $\eta(0)$ to $\eta\left(s_{0}\right)$ is contained in the $n(L)$-neighborhood of $\eta\left[0, s_{0}\right]$ and hence its canonical modifications do not pass through $A$. Similarly, a canonical modification of a level- $p$ hierarchy path connecting $\eta\left(t_{0}\right)$ to $\eta(k)$ does not pass through $A$. By the assumption (1), and the properties of hierarchy paths, this implies that

$$
\operatorname{diam}\left(\pi_{Y}\left(\eta\left(s_{0}\right) \cup \eta\left(t_{0}\right)\right)\right) \geq L(4 n(L)+10) .
$$

The distance in $\mathcal{A}(p)$ between $\eta\left(s_{0}\right), \eta\left(t_{0}\right)$ is at most $2 n(L)+3$, and hence since $\eta$ is an $L$-quasigeodesic, the length of the segment $\eta\left[s_{0}, t_{0}\right]$ is at most $L(2 n(L)+4)$. Then the length of a canonical modification $\widetilde{\eta}[s, t]$ of $\eta\left[s_{0}, t_{0}\right]$ is at most $L(4 n(L)+8)$. Now if $c, d$ are disjoint simple closed curves which intersect $Y$ then the diameter of $\pi_{Y}(c \cup d)$ is at most one. Thus if $\tilde{\eta}(\ell)$ intersects $Y$ for all $\ell$ then

$$
\operatorname{diam}\left(\pi_{Y}(\widetilde{\eta}(s) \cup \widetilde{\eta}(t))\right)=\operatorname{diam}\left(\pi_{Y}\left(\eta\left(s_{0}\right) \cup \eta\left(t_{0}\right)\right)\right) \leq L(4 n(L)+8),
$$

which is a contradiction.

This completes the induction step and proves Proposition 3.1.

The arguments in [4] can now be used without modification to identify the Gromov boundary of $\mathcal{N C}(S, 1)$. To this end let $\mathcal{L}$ be the set of all geodesic laminations on $S$ equipped with the coarse Hausdorff topology. In this topology, a sequence $\left(v_{i}\right)$ converges to $v$ if any limit in the usual Hausdorff topology of a convergent subsequence contains $v$ as a sublamination.

For each thick subsurface $X$ of $S$ let $\mathcal{L}(X) \subset \mathcal{L}$ be the set of all minimal geodesic laminations which fill up $X$, equipped with the coarse Hausdorff topology. We have the following corollary.

Corollary 3.10 The Gromov boundary of $\mathcal{N C}(S, 1)$ equals $\bigcup_{X} \mathcal{L}(X)$ equipped with the coarse Hausdorff topology.

\section{Proof of the Theorem}

In this section we consider an oriented surface $S$ of genus $g \geq 2$ with $m \geq 0$ punctures. In the introduction we defined for $n \geq 1$ the graph $\mathcal{N C}(S, n)$ of nonseparating multicurves in $S$ with $n$ components. Our goal is to show the following theorem. 
Theorem 4.1 For $n<g / 2+1$ the graph $\mathcal{N C}(S, n)$ is hyperbolic.

The case $n=1$ is just Proposition 3.1. For $2 \leq n<g / 2+1$ we use induction on $n$ similar to the arguments in the proof of Proposition 3.1. There are no new tools needed, however all the constructions have to be adjusted to the situation at hand.

We begin by describing an electrification of the graph $\mathcal{N C}(S, n)$. First, for a nonseparating $(n-1)$-multicurve $v \in \mathcal{N C}(S, n-1)$ let $H_{v}$ be the complete subgraph of $\mathcal{N C}(S, n)$ whose vertex set consists of all nonseparating $n$-multicurves containing $v$. We have the following lemma.

Lemma 4.2 There is a natural graph isomorphism $H_{v} \rightarrow \mathcal{N C}(S-v, 1)$.

Proof If $\beta \in H_{v}$ is any nonseparating $n$-multicurve containing $v$ then $\beta-v$ is a nonseparating simple closed curve in $S-v$. If $\beta, \beta^{\prime} \in H_{v}$ are connected by an edge then the nonseparating simple closed curves $\beta-v$ and $\beta^{\prime}-v$ are disjoint and hence they are connected in $\mathcal{N C}(S-v, 1)$ by an edge.

Vice versa, the union with $v$ of any nonseparating simple closed curve $c$ in $S-v$ is a nonseparating multicurve in $H_{v}$. If $c^{\prime} \subset S-v$ is nonseparating and disjoint from $c$ then $v \cup c$ and $v \cup c^{\prime}$ are connected by an edge in $H_{v}$.

This shows the lemma.

Let $\mathcal{H}=\left\{H_{v} \mid v \in \mathcal{N C}(S, n-1)\right\}$.

Lemma 4.3 We have that $\mathcal{N C}(S, n-1)$ is quasi-isometric to the $\mathcal{H}$-electrification of $\mathcal{N C}(S, n)$.

Proof Let $\mathcal{E}$ be the $\mathcal{H}$-electrification of $\mathcal{N C}(S, n)$. Define a vertex embedding $\Lambda: \mathcal{N C}(S, n-1) \rightarrow \mathcal{E}$ by associating to a nonseparating $(n-1)$-multicurve $c$ any nonseparating $n$-multicurve $\Lambda(c)$ containing $c$. We claim that $\Lambda$ is coarsely $8-$ Lipschitz.

To see this let $c_{0}, c_{1}$ be connected by an edge in $\mathcal{N C}(S, n-1)$. Then $c_{1}$ is obtained from $c_{0}$ by removing a component $a$ from $c_{0}$ and replacing it by a component $b$ disjoint from $c_{0}$.

The union $c_{0} \cup b$ is a multicurve with $n$ components. If this multicurve is nonseparating then we can view it as a vertex $x \in \mathcal{E}$. Since $\Lambda\left(c_{0}\right)$ is a nonseparating $n$-multicurve containing $c_{0}$, the distance in $\mathcal{E}$ between $\Lambda\left(c_{0}\right)$ and $x$ equals at most 2. Similarly, the distance in $\mathcal{E}$ between $\Lambda\left(c_{1}\right)$ and $x$ is at most 2 and hence the distance in $\mathcal{E}$ between $\Lambda\left(c_{0}\right)$ and $\Lambda\left(c_{1}\right)$ is at most four. 
If $c_{0} \cup b$ is not nonseparating then $a \cup b$ is a bounding pair in $S-\left(c_{0}-a\right)=S-\left(c_{1}-b\right)$. Choose a nonseparating simple closed curve $\omega \in S-\left(c_{0}-a\right)$ which is disjoint from $a \cup b$ so that both $c_{0} \cup \omega$ and $c_{1} \cup \omega$ are nonseparating. Such a curve exists since the genus $g-n+2$ of $S-\left(c_{0}-a\right)$ is at least three. Apply the argument from the previous paragraph to the nonseparating $(n-1)$-multicurves $c_{0},\left(c_{0}-a\right) \cup \omega$ and to $\left(c_{0}-a\right) \cup \omega, c_{1}$. We conclude that the distance in $\mathcal{E}$ between $\Lambda\left(c_{0}\right)$ and $\Lambda\left(c_{1}\right)$ is at most eight.

On the other hand, a map which associates to a vertex $x \in \mathcal{N C}(S, n) \subset \mathcal{E}$ an $(n-1)-$ multicurve contained in $x$ is a coarsely Lipschitz coarse inverse of $\Lambda$. Thus indeed $\Lambda$ is a quasi-isometry.

By Lemma 4.2, for each vertex $v \in \mathcal{N C}(S, n-1)$ the complete connected subgraph $H_{v}$ of $\mathcal{N C}(S, n)$ is quasi-isometric to the hyperbolic graph $\mathcal{N C}(S-v, 1)$. However, by the results in Section 3, the graph $\mathcal{N C}(S-v, 1)$ is in general not quasi-isometric to the curve graph of $S-v$. Thus controlling distances in these subgraphs via subsurface projection is not immediate. Moreover, for a subsurface $X$ of $S$ of genus $g-n+1$ there are in general many different nonseparating $(n-1)$-multicurves disjoint from $X$.

To resolve this problem we use exactly the strategy from Section 3. Namely, we introduce intermediate graphs $\mathcal{N C}(S, n, p)(p \geq 1)$ which are defined as follows. The vertices of $\mathcal{N C}(S, n, p)$ are nonseparating $n$-multicurves. Two such multicurves $v_{0}, v_{1}$ are connected by an edge of length one if $\hat{v}=v_{0} \cap v_{1}$ is an $(n-1)$-multicurve and if the nonseparating simple closed curves $a=v_{0}-\hat{v}$ and $b=v_{1}-\hat{v}$ are connected by an edge in the graph $\mathcal{A}(S-\widehat{v}, p)$.

The strategy is now to deduce hyperbolicity of $\mathcal{N C}(S, n, 1)$ from hyperbolicity of $\mathcal{N C}(S, n-1)$, and for $p \geq 2$ to deduce hyperbolicity of $\mathcal{N C}(S, n, p)$ from hyperbolicity of $\mathcal{N C}(S, n, p-1)$.

For a nonseparating $(n-1)$-multicurve $v \in \mathcal{N C}(S, n-1)$ let $H_{v}(1)$ be the complete subgraph of $\mathcal{N C}(S, n, 1)$ whose vertex set consists of all nonseparating $n$-multicurves containing $v$. Define moreover

$$
\mathcal{H}(1)=\left\{H_{v}(1) \mid v\right\}
$$

The following is immediate from the reasoning in Lemmas 4.2 and 4.3.

Lemma 4.4 (i) There is a natural graph isomorphism $H_{v}(1) \rightarrow \mathcal{A}(S-v, 1)$.

(ii) We have that $\mathcal{N C}(S, n-1)$ is quasi-isometric to the $\mathcal{H}(1)$-electrification of $\mathcal{N C}(S, n, 1)$. 
Our goal is to apply Theorem 2.3 to the family $\mathcal{H}(1)$ of subgraphs of $\mathcal{N C}(S, n, 1)$ to deduce hyperbolicity of $\mathcal{N C}(S, n, 1)$ from hyperbolicity of $\mathcal{N C}(S, n-1)$. To this end we have to check that the assumptions in the theorem are satisfied.

For $v \neq \zeta \in \mathcal{N C}(S, n-1)$, the vertex set of the intersection $H_{v}(1) \cap H_{\zeta}(1)$ is the set of all nonseparating $n$-multicurves which contain both $v$ and $\zeta$ and hence it consists of at most one point. Thus $\mathcal{H}(1)$ is bounded.

By the first part of Lemma 4.4 and by Lemma 3.2, for every $v \in \mathcal{N C}(S, n-1)$ the graph $H_{v}(1)$ is $\delta$-hyperbolic for a number $\delta>0$ which does not depend on $v$.

The final step is the verification of the bounded penetration property, which is more involved.

Let $\mathcal{E}$ be the $\mathcal{H}(1)$-electrification of $\mathcal{N C}(S, n, 1)$. Let $\beta:[0, k] \rightarrow \mathcal{E}$ be an efficient simplicial quasigeodesic. If the integer $i<k$ is such that $\beta(i), \beta(i+1) \in \mathcal{N C}(S, n, 1)$ then $\beta(i)$ and $\beta(i+1)$ are $n$-multicurves, and $v=\beta(i) \cap \beta(i+1)$ is an $(n-1)-$ multicurve such that $\beta(i), \beta(i+1) \in H_{v}(1)$. If $\beta(i)=v_{v}$ is a special vertex defined by an $(n-1)$-multicurve $v$ then $\beta(i-1), \beta(i+1) \in H_{v}(1)$.

As in Section 2, an enlargement of $\beta$ is a path $\hat{\beta}:[0, m] \rightarrow \mathcal{N C}(S, n, 1)$ defined as follows. For each $i$ such that $\beta(i)=v_{v}$ for some $v \in \mathcal{N C}(S, n-1)$, replace the arc $\beta[i-1, i+1]$ by a path of the form $j \rightarrow v \cup \zeta(j)$ where $\zeta$ is a simplicial geodesic in $\mathcal{A}(S-v, 1)$ connecting $\beta(i-1)-v$ to $\beta(i+1)-v$.

By induction, we now assume that for every $L>1$ there is a number $\kappa^{\prime}(L)>0$ with the following property. Let $X \subset S$ be a connected subsurface of genus $g / 2<h \leq$ $g-n+1<g$. If $\alpha:[0, \ell] \rightarrow \mathcal{N C}(S, n-1)$ is an $L$-quasigeodesic with the property that $\pi_{X}(\alpha(i)) \neq \varnothing$ for all $i$ then the diameter of $\pi_{X}\left(\bigcup_{i} \alpha(i)\right)$ in the curve graph of $X$ does not exceed $\kappa^{\prime}(L)$. Note that the case $n=2$ holds true by the results in Section 3.

Lemma 4.3 then implies that for every $L>1$ there is a number $\kappa(L)>3 L$ with the following property. Let $\beta:[0, k] \rightarrow \mathcal{E}$ be an efficient $L$-quasigeodesic. If $X \subset S$ is a connected subsurface of genus $g / 2<h \leq g-n+1$ such that the diameter of $\pi_{X}(\beta(0) \cup \beta(k))$ in the curve graph of $X$ is at least $\kappa(L)$, then there is an $(n-1)-$ multicurve $v \in \mathcal{N C}(S, n-1)$ disjoint from $X$, and there is some $i<k$ such that $\beta(i) \in H_{v}(1) \subset \mathcal{N C}(S, n, 1)$.

As in Section 3, an enlargement $\hat{\beta}$ of $\beta$ admits a canonical modification $\tilde{\beta}$ as follows. If $\beta(i)=v \cup a, \beta(i+1)=v \cup b$ are such that the simple closed curves $a, b$ are not disjoint but contained in a thick subsurface $X$ of $S-v$ of Euler characteristic $\chi(X)=\chi(S)+1=\chi(S-v)+1$, then replace the edge $\beta[i, i+1]$ in $\mathcal{N C}(S, n, 1)$ by 
an edge-path $\zeta[j-1, j+1]$ of length two in the space of (not necessarily nonseparating) $n$-multicurves so that $\zeta(j-1)=\beta(i)=v \cup a, \zeta(j+1)=\beta(i+1)=v \cup b$ and that $\zeta(j)=v \cup c$ for a (perhaps separating) simple closed curve $c \subset S-v$ which is disjoint from $a, b$ and $X$.

The following can now be derived from the results in Section 2 and Lemmas 4.2 and 4.3.

Proposition 4.5 (i) The graph $\mathcal{N C}(S, n, 1)$ is hyperbolic.

(ii) For every $L>1$ there are numbers $L^{\prime}>1, \kappa(L)>0$ with the following property. Let $\hat{\beta}:[0, k] \rightarrow \mathcal{N C}(S, n, 1)$ be an enlargement of an efficient $L$-quasigeodesic in $\mathcal{E}$.

(a) We have that $\hat{\beta}$ is an $L^{\prime}$-quasigeodesic in $\mathcal{N C}(S, n, 1)$.

(b) Let $X \subset S$ be a connected subsurface of genus $h \in(g / 2, g-n+1]$ and Euler characteristic $\chi(X)=\chi(S)+1$. If $\operatorname{diam}\left(\pi_{X}(\beta(0) \cup \beta(k))\right) \geq \kappa(L)$ then a canonical modification of $\widehat{\beta}$ passes through the complement of $X$.

Proof By the results in Section 2, by Lemmas 4.2 and 4.3, to show hyperbolicity of $\mathcal{N C}(S, n, 1)$ we only have to show the bounded penetration property for efficient quasigeodesics in $\mathcal{E}$ and the family of subgraphs $\mathcal{H}(1)$.

To this end let $\beta:[0, k] \rightarrow \mathcal{E}$ be an efficient $L$-quasigeodesic in $\mathcal{E}$ with endpoints in $\mathcal{N}(S, n, 1)$ and let $v$ be an element of $\mathcal{N C}(S, n-1)$. Assume that

$$
\operatorname{diam}\left(\pi_{S-v}(\beta(0) \cup \beta(k))\right) \geq 3 \kappa(L),
$$

where $\kappa(L)>3 L$ is the constant determined before the statement of the proposition. Then by the assumption on the properties of the space $\mathcal{N C}(S, n-1), \beta$ passes through $H_{v}(1)$.

The diameter of $H_{v}(1)$ in $\mathcal{E}$ equals two. Thus if $0 \leq i \leq j \leq k$ are the first and last points, respectively, of the intersection of $\beta$ with $H_{v}(1)$, then the length $j-i$ of $\beta[i, j]$ is at most $3 L<\kappa(L)$.

Using again the assumption on $\mathcal{N C}(S, n-1)$, for $\beta[0, i]$ and $\beta[j, k]$ we find that

$$
\operatorname{diam}\left(\pi_{X}(\beta(0) \cup \beta(i))\right) \leq \kappa(L) \text { and } \operatorname{diam}\left(\pi_{X}(\beta(j) \cup \beta(k))\right) \leq \kappa(L)
$$

and therefore $\operatorname{diam}\left(\pi_{X}(\beta(i) \cup \beta(j))\right) \geq \kappa(L)>3 L$.

Hence $\beta[i, j]$ passes through the special vertex $v_{\nu}$. Moreover, the points $\beta(i), \beta(j)$ are of the form $v \cup a(i), v \cup a(j)$ for nonseparating simple closed curves $a(i), a(j) \subset S-v$ such that $\operatorname{diam}\left(\pi_{S-v}(\beta(0) \cup a(i))\right) \leq \kappa(L)$ and $\operatorname{diam}\left(\pi_{X}(\beta(k) \cup a(j))\right) \leq \kappa(L)$. This immediately implies the bounded penetration property for the regions $H_{\nu}(1)$ and completes the proof of hyperbolicity of $\mathcal{N C}(S, n, 1)$. 
The other statements are also immediate from the assumptions on $\mathcal{N C}(S, n-1)$ and from Theorem 2.3.

Our aim now is to show that hyperbolicity of $\mathcal{N C}(S, n, p-1)$ implies hyperbolicity of $\mathcal{N C}(S, n, p)$. To this end we proceed as in Section 3. The proofs are completely analogous to the proofs in Section 3.

Once more, our goal is to apply Theorem 2.3. For this denote, for a connected subsurface $X$ of $S$ of genus $h=g-n+1$ and Euler characteristic $\chi(X)=\chi(S)+p-1$, by $B_{X}$ the complete subgraph of $\mathcal{N C}(S, n, p-1)$ whose vertices consist of all nonseparating $n$-multicurves $v$ which are disjoint from the boundary of $X$. By assumption on the genus of $X$, every nonseparating $n$-multicurve $v$ intersects $X$ and therefore a vertex $v \in B_{X}$ has at least one component which is contained in $X$.

In the next lemma, the assumption $n-1<g / 2$ is used in an essential way.

Lemma 4.6 There is a number $L_{0}>1$ not depending on $X$ such that $B_{X}$ is $L_{0}-$ quasi-isometric to $\mathcal{A}(X, 1)$.

Proof Let $v_{0} \subset S-X$ be a nonseparating ( $\left.n-1\right)$-multicurve. Then for every simple closed nonseparating curve $b \subset X$, the union $v_{0} \cup b$ is a vertex in $\mathcal{N C}(S, n, p)$. Moreover, by Lemma 3.5, two such pairs $v_{0} \cup a, v_{0} \cup b$ are connected by an edge in $\mathcal{N C}(S, n, p)$ if and only if $a, b$ are connected by an edge in $\mathcal{A}(X, 1)$.

Now let $\zeta \in B_{X}$ be arbitrary. Then the number $k$ of components of $\zeta$ contained in $X$ is nonzero. Let $c_{1}, \ldots, c_{k}$ be these components. Then $c_{1} \cup \cdots \cup c_{k}$ is a nonseparating $k$-multicurve contained in $X$.

Choose a nonseparating $n$-multicurve $\zeta^{\prime} \supset c_{1} \cup \cdots \cup c_{k}$ contained in $X$. Such a multicurve exists since $n<h$. By the definition of $B_{X}$, the distance in $B_{X}$ between $\zeta$ and $\zeta^{\prime}$ equals $n-k$. Moreover, the distance in $B_{X}$ between $\zeta^{\prime}$ and a nonseparating $n$-multicurve containing $v_{0}$ equals $n-1$. But this just means that the subspace of $B_{X}$ of nonseparating $n$-multicurves containing $v_{0}$ is coarsely dense. Consequently, associating to a vertex $\zeta \in B_{X}$ a component of $\zeta$ contained in $X$ defines a Lipschitz map $B_{X} \rightarrow \mathcal{A}(X, 1)$.

That this map coarsely does not decrease distances follows from the fact that any vertex $\zeta \in B_{X}$ contains at least one component which is contained in $X$, and adjacent vertices contain components which either are disjoint or contained in a common thick subsurface of $X$ of Euler characteristic $\chi(X)+1$. 
Define a family of subgraphs

$$
\mathcal{B}(p)=\left\{B_{X} \mid X\right\}
$$

of $\mathcal{N C}(S, n, p)$ where $X$ runs through the subsurfaces of $S$ of genus $g-n+1$ and Euler characteristic $\chi(X)=\chi(S)+p-1$. By Lemmas 4.6 and 3.2, each of the graphs $B_{X}$ is quasi-isometric to the curve graph of $X$, in particular it is $\delta$-hyperbolic for a number $\delta>0$ not depending on $X$.

We claim that the family $\mathcal{B}(p)$ is bounded. To this end let $X, Y$ be two subsurfaces of $S$ of the same genus $g-n+1$ and the same Euler characteristic $\chi(S)+p-1$. Let $v \in B_{X} \cap B_{Y}$; then $v$ is a nonseparating $n$-multicurve disjoint from the boundaries of both $X, Y$. Since the genus of $X, Y$ equals $g-n+1$, at least one component of $v$ is contained in $X \cap Y$. However, since $X \neq Y, X \cap Y$ is a proper subsurface of $X$. By Lemmas 4.6 and 3.2, $B_{X}$ is quasi-isometric to the curve graph of $X$ and hence the diameter of $B_{X} \cap B_{Y}$ is uniformly bounded.

Lemma 4.6 shows that each of the graphs $B_{X}$ is $\delta$-hyperbolic for a number $\delta>0$ not depending on $X$. Moreover, by the definition of the graphs $\mathcal{N C}(S, n, p)$, the graph $\mathcal{N C}(S, n, p-1)$ is quasi-isometric to the $\mathcal{B}$-electrification of $\mathcal{N C}(S, n, p-1)$. Thus for an application of Theorem 2.3, we are left with showing the bounded penetration property.

However, this property follows by the induction assumption on subsurface projection and Lemma 4.6.

Example The following example was independently observed by Tarik Aougab and Saul Schleimer and shows that the bound $n<g / 2+1$ in the Theorem is sharp.

Namely, let $S$ be a closed surface of genus 4 and let $d$ be a separating simple closed curve which decomposes $S$ into two surfaces $X_{1}, X_{2}$ of genus 2 with one boundary component. Let $\phi_{i}$ be a pseudo-Anosov element in the mapping class group of $X_{i}$ and let $a_{i}$ be a nonseparating simple closed curve in $X_{i}(i=1,2)$. Let moreover $c$ be a nonseparating simple closed curve which is disjoint from $a_{i}$ and intersects $d$ in two points so that $a_{1}, a_{2}, c$ defines a nonseparating 3-multicurve $v$.

For all $k, \ell \in \mathbb{Z}$ the pair $\left(\phi_{1}^{k}, \phi_{2}^{\ell}\right)$ defines a reducible mapping class for $S$, moreover it is easy to see that the distance in $\mathcal{N C}(S, 3)$ between $v$ and $\left(\phi_{1}^{k}, \phi_{2}^{\ell}\right) v$ is comparable to $k+\ell$. The reason is that the subsurface projection of any nonseparating 3 -multicurve in $S$ into each of the subsurfaces $X_{1}, X_{2}$ does not vanish and therefore the distance in $\mathcal{N C}(S, 3)$ between $v$ and $\left(\phi_{1}^{k_{1}}, \phi_{2}^{k_{2}}\right) v$ is bounded from below by a constant multiple of the distance in the curve graph of $X_{i}$ between $a_{i}$ and $\phi_{i}^{k_{i}} a_{i}(i=1,2)$. But this just means that $\mathcal{N C}(S, 3)$ contains a quasi-isometrically embedded $\mathbb{R}^{2}$. 
Define a subsurface $Y$ of $S$ to be $n$-heavy if the genus of $Y$ is at least $g-n+1$. Let $\mathcal{L}(Y)$ be the set of all minimal geodesic laminations which fill up $Y$. Similarly to Corollary 3.10 we have the following.

Corollary 4.7 The Gromov boundary of $\mathcal{N C}(S, n)$ equals $\bigcup_{Y} \mathcal{L}(Y)$ equipped with the coarse Hausdorff topology, where $Y$ passes through the $n$-heavy subsurfaces of $S$.

Remark It seems that the main result in this note can also be obtained with the tools developed in [8]. To the best of our knowledge, these tools do not have any obvious advantage over the tools we used.

\section{References}

[1] T Aougab, Uniform hyperbolicity of the graphs of curves arXiv:1212.3160 To appear in Geom. \& Topol.

[2] B Bowditch, Uniform hyperbolicity of the curve graphs, preprint (2012) To appear in Pacific J. Math.

[3] M Clay, K Rafi, S Schleimer, Uniform hyperbolicity of the curve graph via surgery sequences arXiv:1302.5519

[4] U Hamenstädt, Geometry of graphs of discs in a handlebody arXiv:1101.1843

[5] S Hensel, P Przytycki, R C H Webb, Slim unicorns and uniform hyperbolicity for arc graphs and curve graphs arXiv:1301.5577 To appear in J. Euro. Math. Soc.

[6] H A Masur, Y N Minsky, Geometry of the complex of curves, I: Hyperbolicity, Invent. Math. 138 (1999) 103-149 MR1714338

[7] H A Masur, Y N Minsky, Geometry of the complex of curves, II: Hierarchical structure, Geom. Funct. Anal. 10 (2000) 902-974 MR1791145

[8] H Masur, S Schleimer, The geometry of the disk complex, J. Amer. Math. Soc. 26 (2013) 1-62 MR2983005

Mathematisches Institut, Universität Bonn

Endenicher Allee 60, D-53115 Bonn, Germany

ursula@math.uni-bonn.de

Received: 20 April 2013 Revised: 29 September 2013 